\title{
Postoperative oscillatory brain activity as an add-on prognostic marker in diffuse glioma
}

\author{
Vera Belgers ${ }^{1,2}$. Tianne Numan ${ }^{1,2} \cdot$ Shanna D. Kulik ${ }^{1,2} \cdot$ Arjan Hillebrand $^{3} \cdot$ Philip C. de Witt Hamer $^{2,4}$. \\ Jeroen J. G. Geurts ${ }^{1}$. Jaap C. Reijneveld ${ }^{2,5}$. Pieter Wesseling ${ }^{2,6} \cdot$ Martin Klein $^{2,7}$. Jolanda Derks ${ }^{1,2} \cdot$ Linda Douw $^{1,2,8}$
}

Received: 28 October 2019 / Accepted: 27 December 2019 / Published online: 17 January 2020

(c) The Author(s) 2020

\begin{abstract}
Introduction Progression-free survival (PFS) in glioma patients varies widely, even when stratifying for known predictors (i.e. age, molecular tumor subtype, presence of epilepsy, tumor grade and Karnofsky performance status). Neuronal activity has been shown to accelerate tumor growth in an animal model, suggesting that brain activity may be valuable as a PFS predictor. We investigated whether postoperative oscillatory brain activity, assessed by resting-state magnetoencephalography is of additional value when predicting PFS in glioma patients.

Methods We included 27 patients with grade II-IV gliomas. Each patient's oscillatory brain activity was estimated by calculating broadband power $(0.5-48 \mathrm{~Hz})$ in 56 epochs of $3.27 \mathrm{~s}$ and averaged over 78 cortical regions of the Automated Anatomical Labeling atlas. Cox proportional hazard analysis was performed to test the predictive value of broadband power towards PFS, adjusting for known predictors by backward elimination.

Results Higher broadband power predicted shorter PFS after adjusting for known prognostic factors ( $\mathrm{n}=27$; HR 2.56 (95\% confidence interval (CI) 1.15-5.70); $p=0.022$ ). Post-hoc univariate analysis showed that higher broadband power also predicted shorter overall survival (OS; $\mathrm{n}=38$; HR 1.88 (95\% CI 1.00-3.54); $p=0.038$ ).

Conclusions Our findings suggest that postoperative broadband power is of additional value in predicting PFS beyond already known predictors.
\end{abstract}

Keywords Glioma $\cdot$ Progression-free survival $\cdot$ Overall survival $\cdot$ Magnetoencephalography $($ MEG) $\cdot$ Beamforming

Electronic supplementary material The online version of this article (https://doi.org/10.1007/s11060-019-03386-7) contains supplementary material, which is available to authorized users.

Linda Douw

1.douw@amsterdamumc.nl

1 Anatomy \& Neurosciences, Amsterdam Neuroscience, Amsterdam UMC, Vrije Universiteit Amsterdam, De Boelelaan 1117, $1081 \mathrm{HV}$ Amsterdam, Netherlands

2 Brain Tumor Center, Cancer Center Amsterdam, Amsterdam UMC, Vrije Universiteit Amsterdam, De Boelelaan 1117, 1081 HV Amsterdam, Netherlands

3 Clinical Neurophysiology and MEG Center, Amsterdam Neuroscience, Amsterdam UMC, Vrije Universiteit Amsterdam, De Boelelaan 1117, 1081 HV Amsterdam, Netherlands

4 Neurosurgery, Amsterdam Neuroscience, Amsterdam UMC, Vrije Universiteit Amsterdam, De Boelelaan 1117, 1081 HV Amsterdam, Netherlands
5 Neurology, Amsterdam Neuroscience, Amsterdam UMC, Vrije Universiteit Amsterdam, De Boelelaan 1117, 1081 HV Amsterdam, Netherlands

6 Pathology, Amsterdam Neuroscience, Amsterdam UMC, Vrije Universiteit Amsterdam, De Boelelaan 1117, 1081 HV Amsterdam, Netherlands

7 Medical Psychology, Amsterdam Neuroscience, Amsterdam UMC, Vrije Universiteit Amsterdam, De Boelelaan 1117, 1081 HV Amsterdam, Netherlands

8 Department of Radiology, Athinoula A. Martinos Center for Biomedical Imaging, Massachusetts General Hospital, 149 13th street, Charlestown, MA, USA 


\section{Introduction}

Glioma prognosis is currently determined by factors such as molecular tumor subtype, tumor grade, performance status and age [1]. Still, there is a large variability in progressionfree and overall survival (PFS and OS, respectively) that cannot be explained by these prognostic factors [2,3]. An accurate prognosis is essential for medical decision-making [4]. In order to develop better prognostics, we need to expand our current knowledge of factors that contribute to tumor growth and progression.

One proposed factor associated with glioma growth is neuronal activity. By introducing light-sensitive proteins into the brain, neurons can be activated through light (optogenetics) [5]. This technique was applied in a murine model, showing that the stimulation of neurons promotes glioma growth [6]. This association between neuronal activity and glioma growth is also present without (possibly non-physiological) stimulation through optogenetics: in vitro, spontaneous aberrant neuronal activity is observed even in the earliest stages of glioma [7]. The protein neuroligin-3 (NLGN3) has been suggested to play a key role in glioma growth [8]. NLGN3 is a synaptic adhesion molecule and is important for normal synaptic function and brain plasticity [8]. In the murine model, increased neuronal activity promotes the cleavage and secretion of NLGN3, which then triggers mitosis-and thereby tumor growth - in gliomas [8].

Neuronal activity in patients may be assessed non-invasively using magnetoencephalography (MEG) or electroencephalography (EEG). MEG records the magnetic fields induced by synchronous postsynaptic neuronal activity and, unlike EEG, is not perturbed by the skull and scalp $[9,10]$. The MEG recordings can be projected to sourcespace, resulting in estimated time series of localized neuronal activity [11]. 'Power' is the squared amplitude of the time series and can be analyzed within particular frequency bands or across all frequencies (broadband power; see Fig. 1). Broadband power best correlates with neuronal spiking patterns in patients undergoing both non-invasive neurophysiological measurements as well as single-cell recordings using intracranially placed electrodes [12]. We recently reported on the association between broadband power and PFS in twenty-four newly-diagnosed glioma patients: lower preoperative broadband power predicted longer PFS [13]. While this suggests that broadband power may be a useful prognostic tool in glioma, prognosis based on information after resection might be more reliable than preoperatively measured broadband power. Also, postoperative broadband power can be combined with information on molecular subtype and histology, which are important prognosis predictors. Additionally, postoperative broadband power might logistically be easier to obtain.

To gain a deeper understanding of predictors of PFS in the postoperative clinical setting, we investigated whether higher postoperative broadband power predicts shorter PFS in a cohort of glioma patients. For clinical relevance, we adjusted for already known predictors thereby investigating the added value of broadband power as a predictor. As a post-hoc analysis, we investigated the relationship between broadband power and OS.

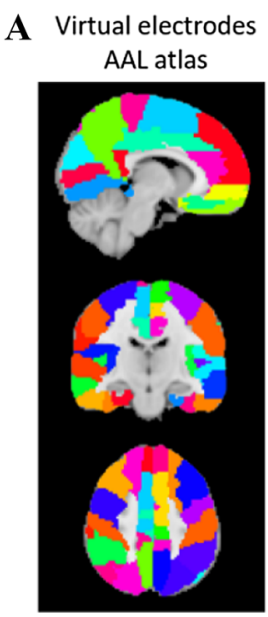

B

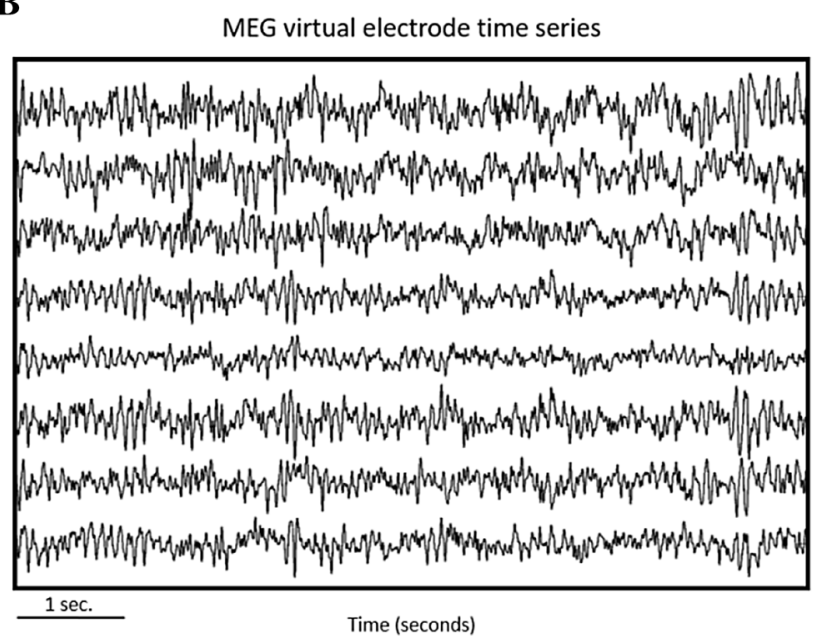

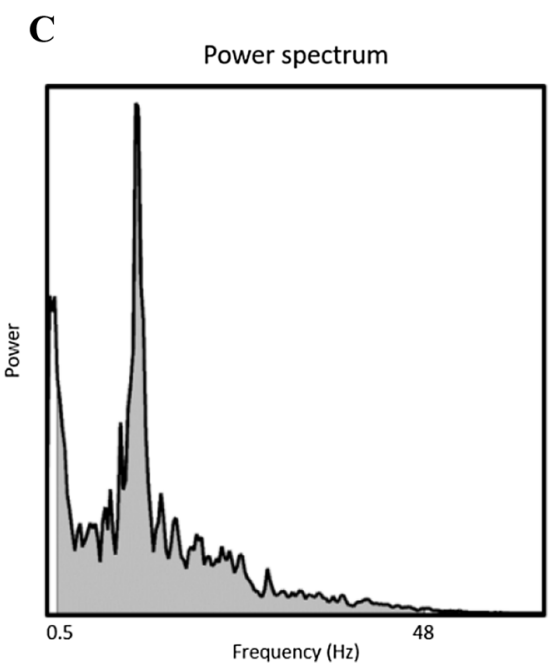

Fig. 1 Broadband power calculated from magnetoencephalography time series: a Time series were estimated, using beamforming, for virtual electrodes placed at the centroids of regions in the automated anatomical labeling (AAL) atlas. b Exemplar broadband time series. c The power spectrum for each time series was obtained using a fast Fourier transform, and then averaged. Broadband power was calculated as the area under the curve between 0.5 and $48 \mathrm{~Hz}$ (grey surface) 


\section{Methods}

\section{Patients}

This retrospective study cohort, which combined several datasets of patients previously reported on, consisted of de novo glioma patients who underwent tumor resection at the Brain Tumor Center Amsterdam [part of Amsterdam University Medical Center, location Vrije Universiteit (VUmc)] between 2010 and 2017 [13, 14]. Inclusion criteria for these studies were (1) age over 17 years, (2) histopathologically confirmed glioma grade II or higher (as defined by the 2016 classification of the World Health Organization [1]), (3) tumor resection as part of their treatment, and (4) no history of neurologic or psychiatric disease. For the current investigation, only patients were included who had undergone a resting-state MEG after tumor resection. Molecular markers (isocitrate dehydrogenase (IDH) mutation and $1 \mathrm{p} 19 \mathrm{q}$ codeletion) were determined in the context of routine clinical care, and therefore not available for all patients [1].

A cohort of healthy controls, previously described in the setting of studies on multiple sclerosis, was used to normalize indices of broadband power $[15,16]$. The healthy subjects were group-level matched to the glioma patients with respect to age, as we know this can affect brain activity $[17,18]$. Data collection in both patients and healthy controls was approved by the ethical review board of the VUmc. Informed consent was obtained before participation.

In the glioma patients, PFS was defined as the time (in weeks) between the MEG recording and the date of radiological or clinical progression, as determined by a multidisciplinary tumor board including a neuroradiologist, neurosurgeon, radiation oncologist, medical oncologist and a neurologist. Patients without progression in the follow-up period (until December 2018) were censored after the last contact.

\section{Magnetoencephalography}

MEG data were obtained using a 306-channel wholehead system (Elektra Neuromag Oy, Helsinki, Finland) with a sampling frequency of $1250 \mathrm{~Hz}$. Brain activity was recorded for $5 \mathrm{~min}$ in a magnetically shielded room (VacuumSchmelze GmbH, Hanua, Germany) during an eyes-closed resting-state in supine position. Patients were instructed to lay still, keep their eyes closed and to stay awake. From the raw data, malfunctioning channels were excluded after visual inspection and artefacts were filtered offline using the temporal extension of Signal Space
Separation in MaxFilter software (Elektra Neuromag Oy, version 2.2.15) [19, 20]. A 3D digitizer (Fastrak, Polhelmus, Colchester, VT, USA) was used to digitize four of five head localization coils and scalp shape. These data were co-registered to the patient's anatomical MRI using a surface-matching approach, to enable projection to the anatomical space. In the 78 cortical regions of the Automated Anatomical Labeling (AAL) atlas, time series were reconstructed through beamforming (see [21-23] for details) and then divided into consecutive epochs (time segments) of $3.27 \mathrm{~s}$ [23]. For each patient, 56 artifactfree epochs were included for analysis, 52 epochs for the healthy controls [19]. No detrending or downsampling was performed, all analyses were performed on data with a sample frequency of $1250 \mathrm{~Hz}$. A fast Fourier transform was performed to obtain the power spectrum for each epoch, and broadband power $(0.5-48 \mathrm{~Hz})$ was calculated. Next, broadband power was averaged over all epochs and all regions per subject. These calculations were performed using Matlab (Mathworks, Natick, MA, USA, version R2012a). The broadband power values were converted to z-scores based on the mean and standard deviation of the healthy subjects.

\section{Statistical analyses}

Data were analyzed using IBM SPSS Statistics for Windows (IBM Corp., Armonk, NY, USA, version 22.0.0.0). P-values smaller than 0.05 were considered statistically significant. Group differences between healthy controls and patients were assessed with Student's t tests for continuous variables and $\chi^{2}$-test for categorical data.

To test our hypothesis on the additional prognostic value of postoperative broadband power towards PFS, we created a Cox proportional hazards model. We adjusted this analysis for age (continuous), Karnofsky performance score (KPS; ordinal), presence of epilepsy (dichotomous), WHO tumor grade (grade II or III/IV) and IDH-mutation/1p19q-status (IDH-mutated (IDH-mut) and 1p19q-codeleted, IDH-mut but non-codeleted, or IDH-wildtype (IDH-wt)) [1, 24-29]. Predictors in the Cox proportional hazards model were selected by backward elimination (cutoff $p<0.1$ ). To avoid overfitting, the maximum number of covariates was determined by dividing the number of patients with progression by six [30]. If more covariates would have been selected through backward elimination, then the least significant predictors were to be eliminated. Robustness of the model was assessed by performing leave-one-out analyses, each time repeating the analysis excluding one patient. Kaplan-Meier plots were rendered for visualization.

Finally, we examined whether our current results were driven by the patients who were previously studied in the presurgical phase of their disease [13]. We did this by 
dividing the cohort into two subgroups (previously/not previously included by Derks and colleagues) [13]. Cox univariate analyses were then performed in these two groups separately.

\section{Results}

\section{Participant characteristics}

Forty patients met the initial inclusion criteria. One patient was excluded due to a comorbid non-brain malignancy, receiving chemotherapy during MEG recording and follow-up. Nine patients had to be excluded from the main analysis due to unknown IDH/1p19q status (these patients were included in the exploratory analysis of OS). Three additional patients were excluded due to progression before MEG (Fig. 2). The final cohort consisted of 27 patients (see Fig. 2 and Supplementary Table 1) and 27 matched healthy controls.

In total, 20 patients showed progression during followup, with a median PFS of 35 (range 0-249) weeks (see Table 1 for detailed participant characteristics). There were no differences between patients and healthy controls in terms of broadband power $(t(55)=0.250, p=0.803)$ or age $(t(52)=-1.94, p=0.058)$. Median time between tumor resection and postoperative MEG recording was 19 weeks (range 3-94). Broadband power and time since tumor resection were not correlated (Kendall's $\tau=0.082, p=0.467$ ).

\section{Higher broadband power relates to shorter progression-free survival}

Backward elimination resulted in a model with broadband power, age and IDH/1p19q status as significant predictors. This indicates that broadband power, age and IDH/1p19q status were significantly predictive of PFS, while tumor grade, epilepsy and KPS were not. In this model, increased broadband power predicted significantly shorter PFS with a hazard ratio (HR) of 2.56 ( $p=0.022$; Fig. 3; Table 2). IDHwt tumors had shorter PFS than IDH-mut tumors (HR 52.44; $p=0.001)$, as expected. Contrary to our expectations, older age predicted longer PFS (HR 0.93; $p=0.032$ ). Post-hoc analyses showed that age alone did not significantly predict PFS (HR 0.99; 95\% CI 0.96-1.03; $p=0.737$ ), moreover, no correlation between age and PFS was found $\left(\mathrm{r}_{\mathrm{s}}=0.14\right.$, $p=0.66$ ).

\section{Robustness of results}

In the leave-one-out validation, broadband power was predominantly significant as a predictor of PFS, while revealing statistically insignificant results four times $(p=0.054 ; 0.061$; $0.10 ; 0.11 ; 14.8 \%)$, as was age $(p=0.051 ; 0.072 ; 0.074$; 0.14 ), indicating some model instability. IDH/1p19q status remained a statistically significant predictor in all models.

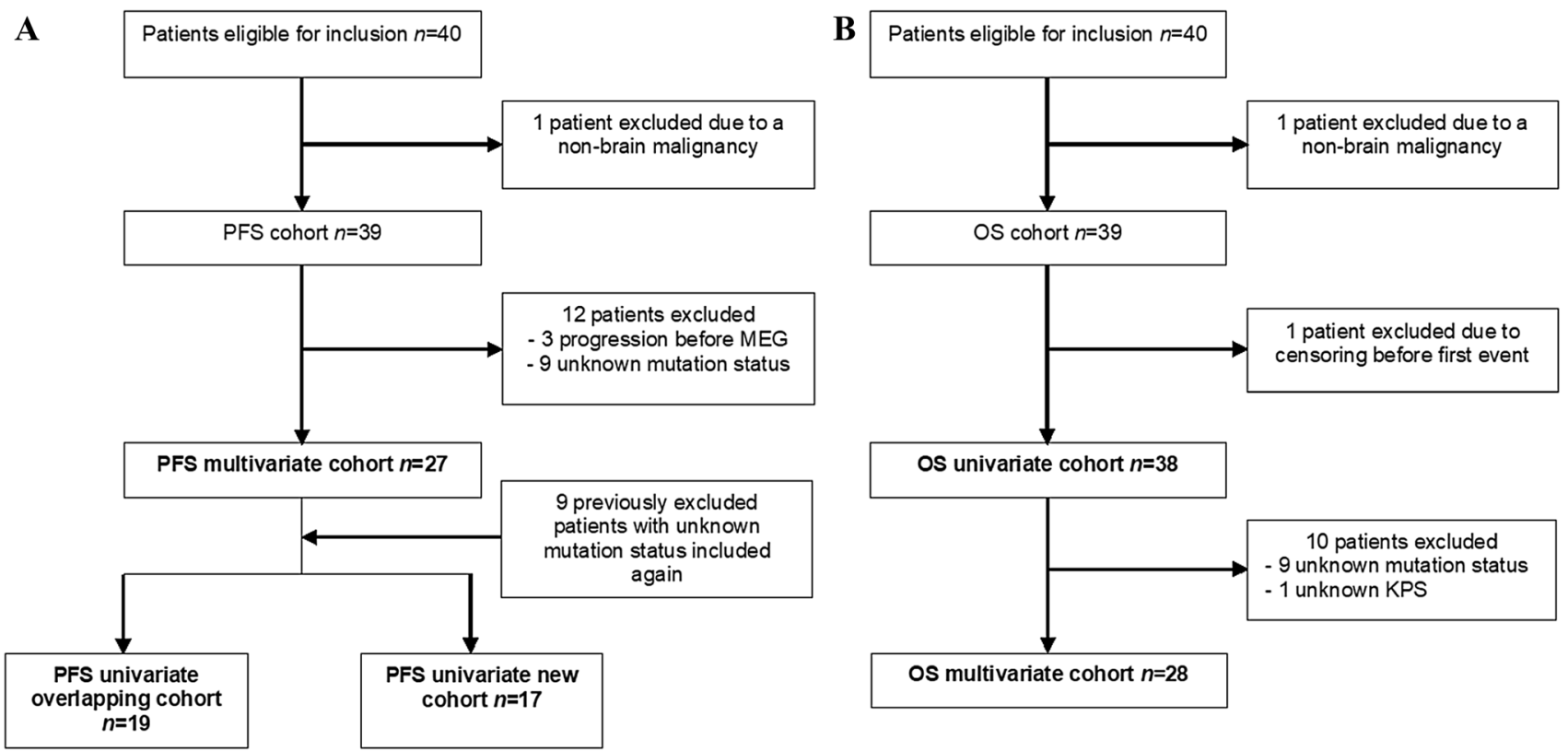

Fig. 2 Flow chart of inclusion for the a PFS cohort (subsequently divided based on whether patients were previously analyzed by Derks et al. [13]) and b OS cohort 
Table 1 Baseline characteristics study cohort

\begin{tabular}{lll}
\hline & Patients (n=27) & $\begin{array}{l}\text { Healthy } \\
\text { controls } \\
(\mathrm{n}=27)\end{array}$ \\
\hline Mean age (SD) & & $46.7(4.7)$ \\
\# Male (\%) & $41.6(12.8)$ & $10(37.0)$ \\
Mean z-score broadband power (SD) & $18(66.7)$ & $0.0(1.0)$ \\
\# Patients with grade II glioma (\%) & $0.0(0.9)$ & \\
\# Patients with grade III glioma (\%) & $13(48.1)$ & \\
\# Patients with grade IV glioma (\%) & $7(25.9)$ \\
IDH/1p19q status & $7(25.9)$ & \\
\# Tumors with IDH-mut/1p19q codeletion (\%) & & \\
\# Tumors with IDH-mut/1p19q non-codeletion (\%) & $6(22.2)$ & \\
\# Tumors with IDH-wt (\%) & $14(51.9)$ & \\
\# Patients with epilepsy (\%) & $7(25.9)$ & \\
\# Patients using AEDs (\%) & $21(77.8)$ & \\
\# Patients treated with radiotherapy (\%) & $21(77.8)$ & \\
\# Patients treated with chemotherapy (\%) & $24(88.9)$ & $17(63.0)$ \\
Median KPS [range] & $100[70-100]$ \\
Median \# weeks between resection and MEG [range] & $22[3-94]$ \\
Median \# weeks PFS or loss to follow-up [range] & $75[0-376]$ \\
Median \# weeks of OS or loss to follow-up (n=38, [range]) & $276[6-372]$ \\
\# Patients with tumor progression (\%) & $20(74.1)$ & \\
\# Patients who died (\%) & $11(40.7)$ & \\
\hline
\end{tabular}

$A E D$ antiepileptic drug, $I D H$ isocitrate dehydrogenase, $K P S$ Karnofsky performance score, $O S$ overall survival, $P F S$ progression-free survival, $S D$ standard deviation, $W T$ wildtype
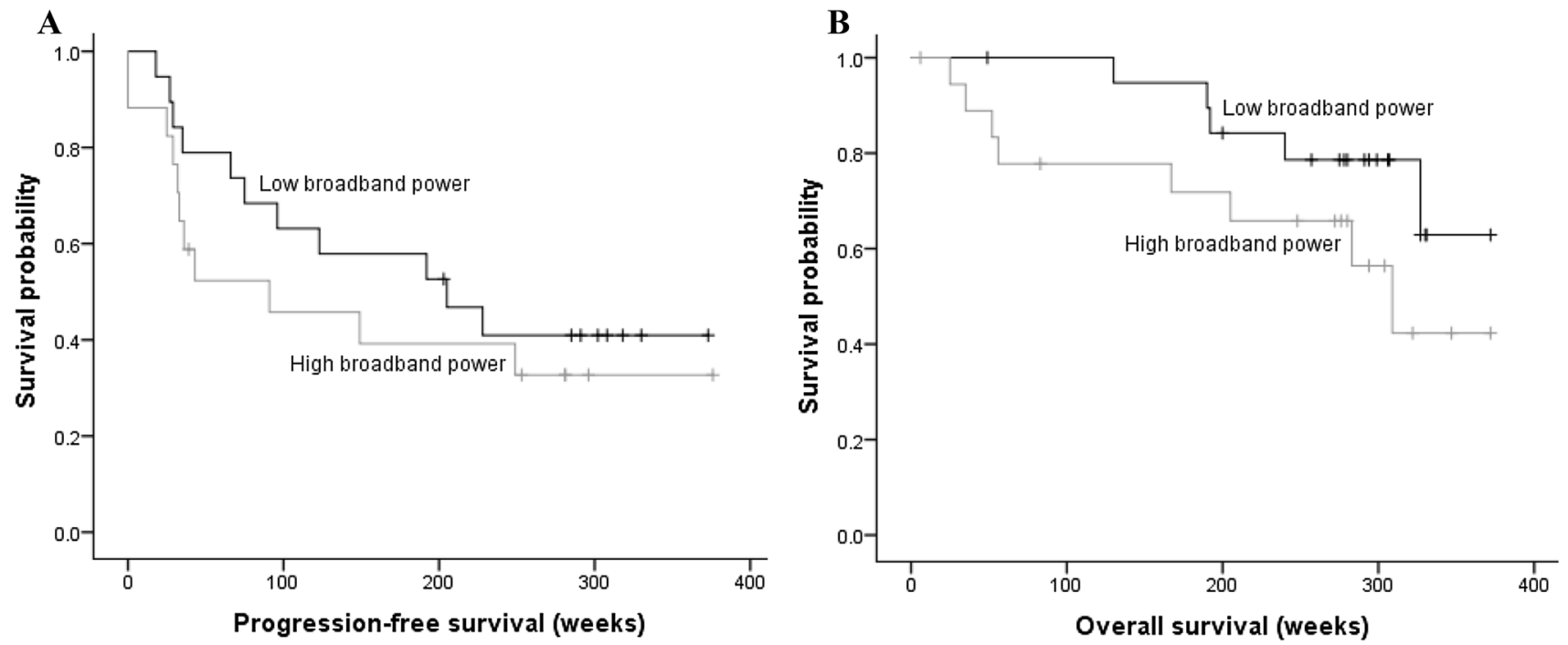

Fig. 3 Kaplan-Meier curves for the a PFS cohort $(n=27)$ and $\mathbf{b}$ OS cohort $(n=38)$ as a function of broadband power, based on a median split in the patient population

\section{Consistency across cohorts}

We split the cohort based on whether patients had previously been analyzed or not (see Fig. 2) [13]. We did not adjust for any other predictors as this was an exploratory univariate analysis. We could therefore include patients with an unknown mutation status, resulting in a cohort of 36 patients (see Fig. 2). This resulted in two groups of roughly equal size: 19 patients overlapped with the cohort of 24 patients analyzed in the presurgical phase by Derks et al., 
Table 2 Cox proportional hazards analyses

\begin{tabular}{llllc}
\hline Model & $\begin{array}{l}\text { Dependent } \\
\text { variable }\end{array}$ & Predictor & HR $(95 \%$ CI $)$ & p-value \\
\hline Multivariate & PFS & Broadband power & $2.56(1.15-5.70)$ & $0.022^{*}$ \\
& & Age & $0.93(0.91-0.97)$ & $0.032^{*}$ \\
& & IDH-mut/codel & Reference & - \\
& & IDH-mut/non-codel & $13.18(1.61-17.02)$ & $0.016^{*}$ \\
Post-hoc univariate & OS & IDH-wt & $52.44(5.28-521.19)$ & $0.001^{*}$ \\
Post-hoc multivariate & OS & Broadband power & $1.94(1.04-3.62)$ & $0.038^{*}$ \\
& & Broadband power & $3.74(1.23-11.36)$ & $0.020^{*}$ \\
& & Age & $1.05(0.97-1.23)$ & 0.30 \\
& & IDH-mut/codel & Reference & - \\
& & IDH-mut/non-codel & $5641.31(0-1.16 \times 1042)^{\mathrm{a}}$ & 0.85 \\
& & IDH-wt & $5.19 \times 1012(0-4.19 \times 1010)^{\mathrm{a}}$ & 0.80 \\
& & KPS & $1.06(0.97-1.15)$ & 0.20 \\
& & Epilepsy & $125.60(2.20-7159.94)$ & $0.019^{*}$ \\
& & Grade II & Reference & - \\
& & Grade III/IV & $0.010\left(0-1.42 \times 10^{36}\right)$ & 0.92 \\
\hline
\end{tabular}

95\% CI 95\% confidence interval, HR hazard ratio, IDH isocitrate dehydrogenase, IDH-mut/codel IDHmutant, $1 p 19 q$ codeleted, IDH-mut/non-codel IDH-mutant, $1 \mathrm{p} 19 \mathrm{q}$ non-codeleted, IDH-wt IDH-wildtype, KPS Karnofsky performance score $* p<0.05$

${ }^{a}$ No people died in the reference group

\begin{tabular}{llllll}
\hline Model & $\begin{array}{l}\text { Dependent } \\
\text { variable }\end{array}$ & Predictor & $\begin{array}{l}\text { Total \# of } \\
\text { patients }\end{array}$ & HR (95\% CI) & p-value \\
\hline Univariate, known cohort & PFS & Broadband power & 19 & $1.61(0.67-3.84)$ & 0.288 \\
Univariate, new cohort & PFS & Broadband power & 17 & $1.95(0.85-4.48)$ & 0.117 \\
\hline
\end{tabular}

95\% CI 95\% confidence interval, $H R$ hazard ratio, $P F S$ progression-free survival
Table 3 Cox proportional hazards analyses after dividing the cohort of progression, or in this case, death [30]. As only thirteen patients had died, the OS multivariate model did not meet this criterium, resulting in an overfitted model. We therefore decided to analyze both a multivariate model to adjust for known predictors, as well as a univariate model to explore the direct relationship between broadband power and OS.

The univariate cohort consisted of 38 patients (see Fig. 2). This model showed broadband power to be predictive of OS (HR 1.94; $p=0.038$; see Table 2). The multivariate cohort was smaller as we excluded 10 patients due to unknown data, resulting in a cohort of 28 patients (see Fig. 2). We selected the most important predictors by performing backward elimination, which meant that in this case, all predictors were included. This model showed broadband power (HR 3.74; $p=0.020$ ) and epilepsy (HR 125.6; $p=0.019$ ) to be significant predictors, see Table 2. It should be noted, however, that no patients with IDH-mutant/1p19q codeleted glioma died within our follow-up time frame, and that the epilepsy subgroup was very small. Both factors possibly attributed to the exorbitant hazard ratios. 
We assessed the robustness of both models by performing leave-one-out analyses, repeating all analyses, each time excluding one patient. This resulted in a univariate nonsignificant model six times $(p=0.051 ; 0.061 ; 0.064 ; 0.076$; $0.093 ; 0.203 ; 15.8 \%)$. The multivariate model revealed statistically non-significant results three times $(p=0.059$; $0.064 ; 0.128 ; 10.7 \%)$.

\section{Exploratory analysis: sensor-level brain activity is not predictive of PFS}

MEG recordings were projected to source-level, resulting in estimated time series of localized neuronal activity [11]. However, sensor-level measurements also provide an estimate of the underlying brain activity. Source-level analysis has a superior spatial resolution and improves the signal-tonoise ratio when techniques such as beamforming are used, thereby improving the estimates of brain activity (see e.g. $[31,32])$. Reconstructing source-level data, however, is a time-consuming task. We therefore explored whether sensorlevel information from gradiometers could also predict PFS. Sensor-level broadband power did not predict PFS in the univariate (HR 1.240; $\mathrm{p}=0.535$ ) or multivariate (HR 1.003; $\mathrm{p}=0.993$ ) analysis (see Supplementary Table 2).

\section{Discussion}

Our results demonstrate that brain activity in glioma patients as measured by MEG is associated with PFS when adjusting for other known predictors (age, tumor grade, KPS, epilepsy and IDH/1p19q status). According to our results, assessment of broadband power could thus improve the current prognosis prediction. Although we did not directly measure tumor growth, these findings also support the hypothesis that higher levels of neuronal activity are associated with faster tumor growth. Activity recorded at the sensor-level did not predict PFS, emphasizing the importance of reconstructing activity at the source-level [31, 32].

Older age was associated with a slightly longer PFS (HR 0.93 ), whereas we expected age to predict a slightly shorter PFS (previously reported HR 1.02-1.06) [28]. This could be due to our small sample size, or to a possible selection bias that resulted in above average vitality in our older patients.

Results from the post-hoc analyses also suggest a relationship between broadband power and overall survival. The univariate model showed broadband power to be predictive of OS, the multivariate model verified this connection. Even though this multivariate model was overfitted and thus had limited statistical power, it suggests that the predictive value of broadband power cannot be explained by other predictors and is an independent predictor of OS.
In our patients, broadband power was assessed after tumor resection, which is a relevant phase of the disease in terms of treatment strategy. Part of the current cohort has been reported on with respect to preoperative broadband power, showing that higher oscillatory activity before tumor resection significantly predicts PFS [13]. Hazard ratios for broadband power were similar in the separate analyses of the previously analyzed cohort and the new cohort, suggesting that our findings are robust, both in terms of time point (before or after tumor resection) and cohorts. Although broadband power did not reach significance in predicting PFS in the two small subgroups (likely due to reduced statistical power), hazard ratios were comparable to those found for the entire cohort. This indicates that the current results were not driven by those patients already included in our previous study but may be seen as a replication and extension of those results.

Broadband power was based on all AAL regions, because Derks et al. showed similar results in the preoperative setting for tumor regions specifically and when including all AAL regions [13]. From a brain network perspective, glioma and the resection of glioma affects the entire brain network and it is therefore reasonable to expect global alterations in broadband power [33, 34].

\section{Clinical implications}

Glioma prognosis and treatment are determined preoperatively and confirmed or adapted postoperatively, based predominantly on histopathology, molecular tumor subtyping, radiological imaging and patient condition. Our results suggest that broadband power could contribute to assessment of prognosis in the postoperative disease phase.

Additionally, broadband power may speculatively be useful in dynamically monitoring disease course. If broadband power is indeed a valid proxy of the neuronal activity that leads to accelerated tumor growth, (forthcoming) tumor progression may conceivably be detected with broadband power before radiological or clinical progression is observable [35]. If so, this would be particularly relevant in the context of pseudoprogression. Pseudoprogression occurs in approximately $10-30 \%$ of patients treated for gliomas, and is difficult to distinguish from real tumor progression [36]. With conventional MRI, an estimated 37\% of diagnosed progressive disease cases are actually (at least partly) pseudoprogression [36]. Consequently, current guidelines discourage diagnosing tumor progression within the first 3 months posttreatment [37]. Due to the aggressive nature of (in particular) glioblastomas, however, progression might very well be seen within these first months, underlining the need for better, non-invasive markers of (early) progression. Larger prospective studies are necessary to investigate the value of broadband power in this context. 
A foreseen barrier towards clinical implementation of our current results is the fact that MEG is costly and not widely available in hospitals around the world, although new technical developments may change this situation rapidly [38]. For now, a more feasible alternative could be to use EEG, as it is widely available and less costly. Although EEG is less accurate and more prone to artifacts than MEG, it might be worth investigating whether EEG also reliably measures broadband neuronal power and predicts PFS.

Even more valuable than improving survival prediction and monitoring, is the possibility of developing new treatment targets based on the mechanism hypothesized to underlie the predictive value of broadband power for PFS. NLGN3 has been suggested to play a key role in glioma growth and might therefore be a viable treatment target [8]. Indeed, gliomas fail to grow in NLGN3 knockout mice, while blocking NLGN3 release prevents tumor growth in animals [6]. However, these inhibitors are currently not suitable for human use. Therefore, a more feasible treatment target might be neuronal activity, which might be reduced through antiepileptic drugs (AEDs) as these could diminish neuronal activity, or through noninvasive inhibitory stimulation using transcranial magnetic stimulation (TMS) or transcranial direct/alternating current stimulation (tDCS/tACS) [35-42]. Clinical trials are necessary to further explore the therapeutic benefits of these targets.

\section{Limitations}

First, although very well-characterized, the study cohort should be considered small, so caution must be applied when interpreting our results. The robustness of the results was evaluated with leave-one-out analyses, showing a 11-16\% model instability, probably due to the low sample size. Nevertheless, our study results are in line with previous findings [13].

Furthermore, the study population does not accurately reflect the general diffuse glioma patient population: our patients generally had prognostically favorable tumors, were relatively young and had high performance status. This selection bias possibly resulted in the surprising finding that older age was associated with longer PFS. Although the effect was small (HR 0.95), it was significant, underlining that we should interpret our findings with caution when extrapolating to the general diffuse glioma population.

Most patients used an AED. Even though AED dosing was titrated in order to achieve seizure freedom and may thus not reach levels necessary for the dose-dependent lowering of neuronal activity, we cannot exclude the possibility that AED use influenced our results [43].
Last, we used broadband activity as a proxy of neuronal firing. Although we did not directly measure neuronal activity, assessment through MEG remains its most accurate noninvasive proxy $[10,12]$.

\section{Conclusions}

Postoperative broadband power as measured with MEG emerges as a valuable addition to currently known predictors of PFS and OS in glioma patients. Larger prospective cohort studies to investigate the relationship between broadband power and tumor growth are needed to translate these findings to clinical practice. Our findings may be a next step towards improving accuracy of glioma prognosis and disease monitoring, and may offer new leads towards new glioma therapy.

Acknowledgements The authors would like to thank Peterjan Ris, Nico Akemann, Ndedi Sijsma, Karin Plugge, Marieke Alting Siberg and Nandy Zwagerman for the MEG acquisitions.

Funding This study was funded by the Dutch Epilepsy Foundation (NEF, Grants 08-08 and 09-09), the Dutch MS Research Foundation (Grant 09-358d), the Netherlands Organization for Scientific Research (NWO-ZonMW Veni Grant 016.146.086) and Society in Science (Branco Weiss Fellowship).

\section{Compliance with ethical standards}

Conflict of interest J. J. Geurts: Research fees from Biogen, Genzyme, Novartis, MerckSerono. President of the Netherland Institute for Health Research and Development. All other authors declare no potential conflicts of interest.

Ethical approval All procedures performed in this study were in accordance with the ethical standards of the institutional research committee and with the 1964 Helsinki declaration and its later amendments or comparable ethical standards. Informed consent was obtained from all individual participants included in the study.

Open Access This article is licensed under a Creative Commons Attribution 4.0 International License, which permits use, sharing, adaptation, distribution and reproduction in any medium or format, as long as you give appropriate credit to the original author(s) and the source, provide a link to the Creative Commons licence, and indicate if changes were made. The images or other third party material in this article are included in the article's Creative Commons licence, unless indicated otherwise in a credit line to the material. If material is not included in the article's Creative Commons licence and your intended use is not permitted by statutory regulation or exceeds the permitted use, you will need to obtain permission directly from the copyright holder. To view a copy of this licence, visit http://creativecommons.org/licenses/by/4.0/. 


\section{References}

1. Louis DN et al (2016) The 2016 World Health Organization Classification of Tumors of the Central Nervous System: a summary. Acta Neuropathol 131:803-820

2. Di Carlo DT, Duffau H, Cagnazzo F, Benedetto N, Morganti R, Perrini P (2018) IDH wild-type WHO grade II diffuse lowgrade gliomas. A heterogeneous family with different outcomes. Systematic review and meta-analysis. Neurosurg Rev. https:// doi.org/10.1007/s10143-018-0996-3

3. Poulen G, Gozé C, Rigau V, Duffau H (2018) Huge heterogeneity in survival in a subset of adult patients with resected, wild-type isocitrate dehydrogenase status, WHO grade II astrocytomas. J Neurosurg 1:1-10

4. Halabi S, Owzar K (2010) The importance of identifying and validating prognostic factors in oncology. Semin Oncol 37(2):1-15

5. Boyden ES, Zhang F, Bamberg E, Nagel G, Deisseroth K (2005) Millisecond-timescale, genetically targeted optical control of neural activity. Nat Neurosci 8(9):1263-1268

6. Venkatesh HS et al (2017) Targeting neuronal activity-regulated neuroligin-3 dependency in high-grade glioma. Nat Publ Gr 549(7673):533-537

7. Savarraj JP, Kelly KC, DeCoster MA (2019) Early glioma is associated with abnormal electrical events in cortical cultures. Med Biol Eng Comput 57:1645

8. Venkatesh HS et al (2015) Neuronal activity promotes glioma growth through neuroligin-3 secretion. Cell 161(4):803-816

9. Baillet $S$ (2017) Magnetoencephalography for brain electrophysiology and imaging. Nat Neurosci 20(3):327-339

10. Lopes da Silva F (2013) EEG and MEG: relevance to neuroscience. Neuron 80(5):1112-1128

11. Hillebrand A, Singh KD, Holliday IE, Furlong PL, Barnes GR (2005) A new approach to neuroimaging with magnetoencephalography. Human Brain Mapp 211(25):199-211

12. Manning JR, Jacobs J, Fried I, Kahana MJ (2009) Broadband shifts in LFP power spectra are correlated with single-neuron spiking in humans. J Neurosci 29(43):13613-13620

13. Derks J, Wesseling P, Carbo EWS, Hillebrand A, Van Dellen E (2018) Oscillatory brain activity associates with neuroligin-3 expression and predicts progression free survival in patients with diffuse glioma. J Neurooncol 140:403-412

14. Derks J et al (2019) Understanding cognitive functioning in glioma patients: the relevance of IDH-mutation status and functional connectivity. Brain Behav 9(4):1-9

15. Tewarie $P$ et al (2014) Structural degree predicts functional network connectivity: a multimodal resting-state fMRI and MEG study. Neuroimage 97:296-307

16. Tewarie $P$ et al (2015) Functional brain networks: linking thalamic atrophy to clinical disability in multiple sclerosis, a multimodal fMRI and MEG study. Human Brain Mapp 36:603-618

17. Mandal PK, Banerjee A, Tripathi M, Sharma A (2018) A comprehensive review of magnetoencephalography (MEG) studies for brain functionality in healthy aging and Alzheimer's disease (AD). Front Comput Neurosci 12:60

18. Ishii $\mathrm{R}$ et al (2018) Healthy and pathological brain aging: from the perspective of oscillations, functional connectivity, and signal complexity. Neuropsychobiology 75(4):151-161

19. Taulu S, Hari R (2009) Removal of magnetoencephalographic artifacts with temporal signal-space separation: demonstration with single-trial auditory-evoked responses. Human Brain Mapp 1534(30):1524-1534

20. Taulu S, Simola J (2005) Spatiotemporal signal space separation method for rejecting nearby interference in MEG measurements Spatiotemporal signal space separation method for rejecting nearby interference in MEG measurements. Phys Med Biol 51:1-10

21. Hillebrand A, Barnes GR, Bosboom JL, Berendse HW, Stam CJ (2012) Frequency-dependent functional connectivity within resting-state networks: an atlas-based MEG beamformer solution. Neuroimage 59(4):3909-3921

22. Hillebrand A et al (2016) Direction of information flow in largescale resting-state networks is frequency-dependent. Proc Natl Acad Sci USA 113(14):3867-3872

23. Tzourio-Mazoyer $\mathrm{N}$ et al (2002) Automated anatomical labeling of activations in SPM using a macroscopic anatomical parcellation of the MNI MRI single-subject brain. Neuroimage 15(1):273-289

24. Zhou H, Tan X, Yang L, Bai HX, Huang X, Xiao B (2016) Prognostic relevance of epilepsy at presentation in lower-grade gliomas. Neuro Oncol 18:1326-1327

25. Gorlia T et al (2013) New validated prognostic models and prognostic calculators in patients with low-grade gliomas diagnosed by central pathology review: a pooled analysis of EORTC/RTOG/ NCCTG phase III clinical trials. Neuro Oncol 15(11):1568-1579

26. Zhao J, Ma W, Zhao H (2014) Loss of heterozygosity 1p/19q and survival in glioma: a meta-analysis. Neuro Oncol 16(1):103-112

27. Sanson $M$ et al (2009) Isocitrate dehydrogenase 1 codon 132 mutation is an important prognostic biomarker in gliomas. J Clin Oncol 27(25):4150-4154

28. Fekete B, Werlenius K, Örndal C, Rydenhag B (2016) Prognostic factors for glioblastoma patients: a clinical population-based study. Acta Neurol Scand 133:434-441

29. Kerkhof M, Vecht CJ (2013) Seizure characteristics and prognostic factors of gliomas. Epilepsia 54(SUPPL. 9):12-17

30. Vittinghoff E, McCulloch CE (2006) Relaxing the rule of ten events per variable in logistic and cox regression. Am J Epidemiol 165(6):710-718

31. Hillebrand A et al (2016) Detecting epileptiform activity from deeper brain regions in spatially filtered MEG data. Clin Neurophysiol 127(8):2766-2769

32. van Klink N, Hillebrand A, Zijlmans M (2016) Identification of epileptic high frequency oscillations in the time domain by using MEG beamformer-based virtual sensors. Clin Neurophysiol 127(1):197-208

33. van Dellen E, Hillebrand A, Douw L, Heimans JJ, Reijneveld JC, Stam CJ (2013) Local polymorphic delta activity in cortical lesions causes global decreases in functional connectivity. Neuroimage 83:524-532

34. Bosma I et al (2009) Disturbed functional brain networks and neurocognitive function in low-grade glioma patients: a graph theoretical analysis of resting-state MEG. Nonlinear Biomed Phys 3:9

35. Filbin MG, Segal RA (2015) How neuronal activity regulates glioma cell proliferation. Neuro Oncol 17(12):1543-1544

36. Thust SC, van den Bent MJ, Smits M (2018) Pseudoprogression of brain tumors. J Magn Reson Imaging. https://doi.org/10.1002/ jmri.26171

37. Wen PY et al (2010) Updated response assessment criteria for high-grade gliomas: response assessment in neuro-oncology working group. J Clin Oncol 28(11):1963-1972

38. Boto E et al (2018) Moving magnetoencephalography towards real-world applications with a wearable system. Nat Publ Gr 555(7698):657-661

39. Lenz M, Vlachos A (2016) Releasing the cortical brake by noninvasive electromagnetic stimulation? rTMS induces LTD of GABAergic neurotransmission. Front Neural Circ 10:1-9

40. Krause B, Márquez-ruiz J, Kadosh RC (2013) The effect of transcranial direct current stimulation: a role for cortical excitation/ inhibition balance? Front Hum Neurosci 7:1-4 
41. Zhang Q et al (2018) Antiepileptic drug of levetiracetam decreases centrotemporal spike-associated activation in rolandic epilepsy. Front Neurosci 12(796):1-9

42. Englund M, Hyllienmark L, Brismar T (2011) Effect of valproate, lamotrigine and levetiracetam on excitability and firing properties of CA1 neurons in rat brain slices. Cell Mol Neurobiol $31: 645-652$

43. Clemens B (2008) Valproate decreases EEG synchronization in a use-dependent manner in idiopathic generalized epilepsy. Seizure $17: 224-233$
Publisher's Note Springer Nature remains neutral with regard to jurisdictional claims in published maps and institutional affiliations. 